\title{
Needle aspiration for treating iatrogenic pneumothorax after cardiac electronic device implantation: a pilot study
}

\author{
Dominika Domokos ${ }^{1} \cdot$ Andras Szabo $^{2} \cdot$ Gyongyver Banhegyi ${ }^{3}$ • Balazs Polgar ${ }^{4} \cdot$ Zsolt Bari $^{4} \cdot$ Peter Bogyi $^{4}$. \\ Istvan Marczell ${ }^{4} \cdot$ Leticia Papp $^{4} \cdot$ Robert Gabor Kiss $^{4} \cdot$ Gabor Zoltan Duray $^{4} \cdot$ Bela Merkely $^{1} \cdot$ Istvan Hizoh $^{1}$
}

Received: 30 May 2019 / Accepted: 15 July 2019/Published online: 24 July 2019

(C) The Author(s) 2019

\begin{abstract}
Purpose Pneumothorax (PTX) following cardiac implantable electronic device procedures is traditionally treated with chest tube drainage (CTD). We hypothesized that, in a subset of patients, the less invasive needle aspiration (NA) may also be effective. We compared the strategy of primary NA with that of primary CTD in a single-center observational study.

Methods Of the 970 procedures with subclavian venous access between January 2016 and June 2018, 23 patients had PTX requiring intervention. Beginning with March 2017, the traditional primary CTD (9 cases) has been replaced by the "NA first" strategy (14 patients). Outcome measures were procedural success rate and duration of hospitalization evaluated both as time to event (log-rank test) and as a discrete variable (Wilcoxon-Mann-Whitney test).

Results Needle aspiration was successful in 8/14 (57.1\%) of the cases (95\% CI 28.9-82.3\%), whereas PTX resolved in all patients after CTD was $9 / 9(100 \%, 95 \%$ CI 66.4-100.0\%, $p=0.0481)$. Regarding length of hospital stay, intention to treat time to event analysis showed no difference between the two approaches $(p=0.73)$. Also, the median difference was not statistically significant $(-2.0$ days, $p=0.17)$. In contrast, per protocol evaluation revealed reduced risk of prolonged hospitalization for NA patients $(p=0.0025)$ with a median difference of -4.0 days $(p=0.0012)$. Failure of NA did not result in a meaningful delay in discharge timing as median difference was 1.5 days $(p=0.28)$.

Conclusions Our data suggest that in a number of patients iatrogenic PTX may be successfully treated with NA resulting in shorter hospitalization without the risk of meaningful discharge delay in unsuccessful cases.
\end{abstract}

Keywords Cardiac implantable electronic device $\cdot$ Complication $\cdot$ Pneumothorax $\cdot$ Chest tube drainage $\cdot$ Needle aspiration

\section{Introduction}

Iatrogenic pneumothorax (PTX) is a major complication of cardiac implantable electronic device (CIED) procedures. The incidence of PTX after CIED implantation varies between 0.7 and $1.7 \%$ according to recent publications [1-3]. It was found to be related to new subclavian venous access site and may be associated with low body mass index (BMI) and

Istvan Hizoh

istvan.hizoh@alumni.uni-heidelberg.de

1 Heart and Vascular Center, Semmelweis University, Budapest 1122, Hungary

2 Department of Anesthesiology and Intensive Care, Semmelweis University, Budapest, Hungary

3 Independent Researcher, Budapest, Hungary

4 Department of Cardiology, Medical Center Hungarian Defense Forces, Budapest, Hungary female gender [1, 4-6]. Pneumothorax requiring intervention is traditionally treated by large-bore chest tube drainage (CTD). Simple pneumothoraces, however, could also be treated successfully with the less invasive catheter aspiration as was shown by Delius et al. in 1989: for needle-induced PTX the success rate was 75\% [7]. Similarly, needle aspiration (NA) has been found to be effective for primary and secondary spontaneous pneumothoraces in recent randomized trials $[8,9]$. Compared with chest tube thoracostomy, needle aspiration is less traumatic and may help to shorten hospital stay thereby reducing costs. Though there is still no general consensus regarding this therapeutic modality, the British Thoracic Society recommended needle aspiration as a firstline therapy for primary spontaneous pneumothorax and as an option for small secondary spontaneous PTX as early as 2010 [10]. Yet, there are no data available about needle aspiration as a treatment option for iatrogenic PTX after CIED implantation. We hypothesized that, at least in a subset of patients, NA might also be effective in this clinical setting. 


\section{Methods}

\subsection{Study design, outcome measures}

Using a prospective registry, we analyzed data of 970 consecutive patients who underwent CIED implantation, upgrade, or revision with at least one new subclavian vein access at the Medical Center Hungarian Defense Forces from January 2016 to June 2018. Of them, in 11 cases $(1.1 \%)$, small pneumothoraces were resolved spontaneously, whereas 23 patients $(2.4 \%)$ had intervention-requiring PTX. The decision about treatment was left to the operator's judgment based on clinical symptoms and imaging by X-ray (for details, see Sect. 2.2). In cases necessitating intervention, traditional chest tube drainage was performed in all patients until March 2017 ("historic" control group, 9 cases), when the institutional protocol changed and primary CTD was replaced by the novel "needle aspiration first" strategy (14 patients). In cases with persistent PTX, thoracostomy has been done secondarily. We estimated procedural success rates and length of hospitalization as efficacy outcome measures. Safety of the procedures was assessed by the occurrence of infection, bleeding, and death of any cause during hospital stay. All four physicians involved in the interventions were high-volume operators. The study was conducted in accordance with the amended Declaration of Helsinki. The protocol was approved by the Institutional Ethics Committee on Human Research of the Medical Center Hungarian Defense Forces (approval number: 280-261/2018). All procedures were carried out with the patients' written permission and all of them gave informed consent to participate in the registry.

\subsection{Needle aspiration}

The institutional standard of care for large pneumothoraces was chest tube drainage with additional Heimlich valve under continuous suction. Since March 2017, with the change of the institutional protocol, needle aspiration was attempted for all PTXs requiring intervention regardless of the size of the PTX. The presence of PTX was checked using chest X-ray (CXR) in all patients. In case of clinical suspicion, CXR was done immediately; otherwise, it was performed the day after CIED implantation. Thus, PTX was detected within $24 \mathrm{~h}$ after the procedure. In addition to traditional interpleural distance measurements, size of the PTX was also estimated as percentage PTX volume according to Collins et al. [11]. The need for intervention was determined by clinical signs and symptoms and/or CXR findings according to the guideline of the British Thoracic Society: visible rim of more than $2 \mathrm{~cm}$ between the lung margin and the chest wall at the level of the hilum [10]. We implemented the checklist and technical protocol published by Pasquier in 2013 [12]. The puncture site was the second intercostal space in the midclavicular line a safe distance from both the generator, which is placed more laterally, and the internal mammary artery, which is located medial to the intended puncture track [13]. All patients received nasal oxygen therapy using a face mask. During the procedure oxygen saturation, heart rate, and blood pressure were monitored. The procedure was carried out under local anesthesia and aseptic conditions using a 16- or 18-gauge over-theneedle cannula, a three-way stopcock, and a $50-\mathrm{mL}$ syringe. The volume of the aspiration was limited to $2500 \mathrm{~mL}$ since larger volumes indicate a persistent air leak requiring chest drain insertion [10]. In these failed needle aspiration cases, CTD was performed immediately. At the end of the intervention, the cannula was removed and a bandage was placed. Success of the procedure was checked by regular chest Xrays 1,6 , and $24 \mathrm{~h}$ following the intervention. The procedure was considered successful if the patient was asymptomatic and there was no residual PTX visible on the subsequent CXRs or it showed marked regression being at most a few millimeters at the apex of the lung. Patients with resolved PTX were discharged, whereas in persistent cases regular chest tube drainage was performed by a thoracic surgeon with continuous suction until no residual PTX was detectable on CXR. After removal of the chest tube, patients were observed for $24 \mathrm{~h}$ before discharge.

\subsection{Statistical analysis}

Continuous baseline parameters were examined for normality with the D'Agostino-Pearson and Shapiro-Wilk tests. As none of the continuous variables showed normal distribution, the exact Wilcoxon-Mann-Whitney test was applied for comparisons. Variables in $2 \times 2$ contingency tables were assessed using Fisher's exact test. We estimated procedural success rates and length of hospital stay as efficacy outcome measures. We analyzed raw data, since the small sample size did not allow the use of multivariable adjustment or propensity score-based techniques. Success rates of the different therapeutic approaches and their uncertainty were calculated using the exact binomial test, whereas Fisher's exact test was performed for their comparison. Length of hospital stay was evaluated as "time to event" by Kaplan-Meier analysis and the exact log-rank test. In addition, it was analyzed as a discrete variable using the exact Wilcoxon-Mann-Whitney test. Besides intention to treat (ITT) and per protocol (PP) analyses, we also investigated the effect of secondary CTD (i.e., NA failure) on discharge timing. A two-tailed $p$ value less than 0.05 was considered statistically significant. Computations were performed with $\mathrm{R}$ version 3.6.0 (R Foundation for Statistical Computing, Vienna, Austria) using the fBasics 3042.89, coin 1.30, rms 5.1-3.1, survival 2.44-1.1, survminer 0.4.4, and ggplot2 3.2.0 packages. 


\section{Results}

\subsection{Patient characteristics}

Baseline demographic, clinical, and procedural characteristics of patients with intervention-requiring pneumothorax or no PTX and comparisons of the two treatment arms are summarized in Table 1. Patients with pneumothorax necessitating intervention had a lower body mass index and were more likely to be female. Demographic, clinical, and procedural characteristics of the two treatment groups were similar, yet coronary artery disease was more common among primary chest tube drainage patients. No safety outcome events (i.e., infection, bleeding, and death of any cause) occurred in any of the treatment arms during the studied period. Anatomically, the majority of the patients in the needle aspiration arm had partial PTX; there was only one massive PTX case with a totally collapsed lung (estimated median percentage PTX volume $39.5 \%$ (interquartile range (IQR) 32.75 to $54.75 \%$ )).

\subsection{Procedural success rates}

Figure 1 shows the study algorithm. Out of 14 attempts, needle aspiration was successful in 8 patients $(57.1 \%, 95 \%$ confidence interval (CI) 28.9 to $82.3 \%$ ), whereas pneumothorax was resolved in all cases after chest tube drainage (9/9 (100\%), 95\% CI 66.4 to $100.0 \%, p=0.0481)$. All patients with failed primary needle aspiration $(6 / 14(42.9 \%), 95 \%$ CI 17.7 to $71.1 \%)$ were treated successfully with secondary CTD.

\subsection{Length of hospital stay, intention to treat analysis}

There was no statistically significant difference in time to event between the two treatment approaches according to the exact log-rank test ( $p=0.73$, Fig. 2, left panel). Similarly, median in hospital stays were comparable: 4.5 days (IQR 2.0 to 6.0 days) in the NA and 6.0 days (IQR 6.0 to 7.0 days) in the CTD group, median difference -2.0 days, $95 \% \mathrm{CI}-4.0$ to 1.0 days, $p=0.17$, exact Wilcoxon-MannWhitney test (Fig. 3, upper panel).

\subsection{Length of hospital stay, per protocol analysis}

In contrast, per protocol evaluation revealed reduced risk of prolonged hospitalization for successful needle aspiration patients $(n=8)$ compared with primary chest tube thoracostomy cases ( $n=9, p=0.0025$, exact log-rank test, Fig. 2, right panel). Also, median length of hospital stays were different:

Table 1 Baseline demographic, clinical, and procedural characteristics

\begin{tabular}{|c|c|c|c|c|c|c|}
\hline \multirow[b]{2}{*}{ Variable } & \multicolumn{3}{|c|}{ Cardiac implantable electronic device procedures } & \multicolumn{3}{|c|}{$\begin{array}{l}\text { Cases with pneumothorax requiring } \\
\text { intervention }\end{array}$} \\
\hline & $\begin{array}{l}\text { No pneumothorax } \\
(n=936)\end{array}$ & $\begin{array}{l}\text { Pneumothorax } \\
\text { requiring } \\
\text { intervention }(n=23)\end{array}$ & $p$ value & $\begin{array}{l}\text { Primary chest } \\
\text { tube } \\
\text { drainage }(n=9)\end{array}$ & $\begin{array}{l}\text { Primary needle } \\
\text { aspiration } \\
(n=14)\end{array}$ & $\begin{array}{l}p \\
\text { value }\end{array}$ \\
\hline Age, median (IQR) (years) & $74.0(66.0-81.0)$ & $77.0(71.0-81.0)$ & 0.25 & $78.0(71.0-88.0)$ & $76.5(71.0-78.8)$ & 0.68 \\
\hline $\begin{array}{l}\text { Body mass index, } \\
\text { median }(\mathrm{IQR})\left(\mathrm{kg} / \mathrm{m}^{2}\right)\end{array}$ & $27.3(24.5-30.9)$ & $24.4(21.9-25.7)$ & $<0.0001$ & $25.4(24.4-26.0)$ & $23.6(21.7-25.3)$ & 0.31 \\
\hline Female & $330(35.3 \%)$ & $13(56.5 \%)$ & 0.0463 & $3(33.3 \%)$ & $10(71.4 \%)$ & 0.10 \\
\hline Hypertension & $786(84.8 \%)$ & $18(78.3 \%)$ & 0.40 & $6(66.7 \%)$ & $12(85.7 \%)$ & 0.34 \\
\hline Diabetes mellitus & $311(33.2 \%)$ & $5(21.7 \%)$ & 0.37 & $3(33.3 \%)$ & $2(14.3 \%)$ & 0.34 \\
\hline $\begin{array}{l}\text { Chronic obstructive pulmonary } \\
\text { disease }\end{array}$ & $149(15.9 \%)$ & $6(26.1 \%)$ & 0.24 & $2(22.2 \%)$ & $4(28.6 \%)$ & 1.00 \\
\hline Coronary artery disease & $299(31.9 \%)$ & $8(34.8 \%)$ & 0.82 & $6(66.7 \%)$ & $2(14.3 \%)$ & 0.02 \\
\hline Coronary artery bypass graft surgery & $92(9.8 \%)$ & $2(8.7 \%)$ & 1.00 & $1(11.1 \%)$ & $1(7.1 \%)$ & 1.00 \\
\hline Ejection fraction $\leq 35 \%$ & $354(37.8 \%)$ & $8(34.8 \%)$ & 0.83 & $4(44.4 \%)$ & $4(28.6 \%)$ & 0.66 \\
\hline Anamnestic malignancy & $112(12.0 \%)$ & $4(17.4 \%)$ & 0.51 & $1(11.1 \%)$ & $3(21.4 \%)$ & 1.00 \\
\hline Anamnestic radiotherapy & $28(3.0 \%)$ & $2(8.7 \%)$ & 0.16 & $0(0.0 \%)$ & $2(14.3 \%)$ & 0.50 \\
\hline Atrial fibrillation/flutter & $344(36.8 \%)$ & $8(34.8 \%)$ & 1.00 & $3(33.3 \%)$ & $5(35.7 \%)$ & 1.00 \\
\hline Emergency device implantation & $254(27.1 \%)$ & $5(21.7 \%)$ & 0.64 & $2(22.2 \%)$ & $3(21.4 \%)$ & 1.00 \\
\hline Multiple subclavian leads & $459(49.0 \%)$ & $12(52.2 \%)$ & 0.83 & $4(44.4 \%)$ & $8(57.1 \%)$ & 0.68 \\
\hline $\begin{array}{l}\text { Operator's daily number of } \\
\text { implantations, median (IQR) }\end{array}$ & $3.0(1.0-4.0)$ & $2.0(1.0-4.0)$ & 0.46 & $2.0(1.0-3.0)$ & $2.0(1.0-4.0)$ & 0.87 \\
\hline $\begin{array}{l}\text { Time at implantation, } \\
\text { median (IQR) (h) }\end{array}$ & $12.0(10.0-15.0)$ & $11.0(8.5-14.5)$ & 0.49 & $10.0(8.0-14.0)$ & $12.0(9.3-15.5)$ & 0.48 \\
\hline
\end{tabular}


Fig. 1 Study algorithm. Between January 2016 and June 2018, 970 patients underwent procedures with at least one new subclavian vein access. Of them, 23 patients had intervention-requiring pneumothorax (PTX) (for details, see text). CIED, cardiac implantable electronic device; CTD, chest tube drainage

\section{Study Algorithm}

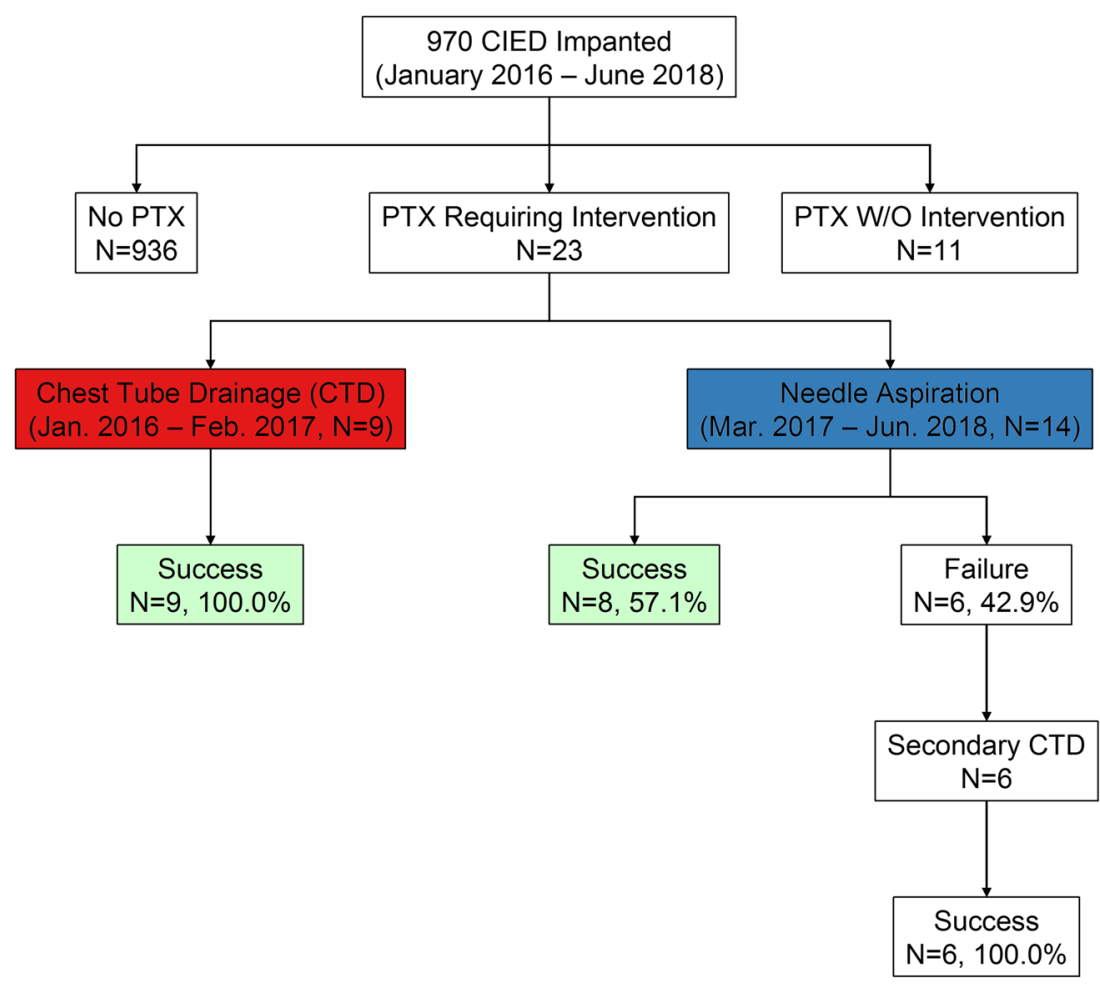

2.0 days (IQR 2.0 to 3.25 days) and 6.0 days (IQR 6.0 to 7.0 days), respectively, median difference -4.0 days, $95 \%$
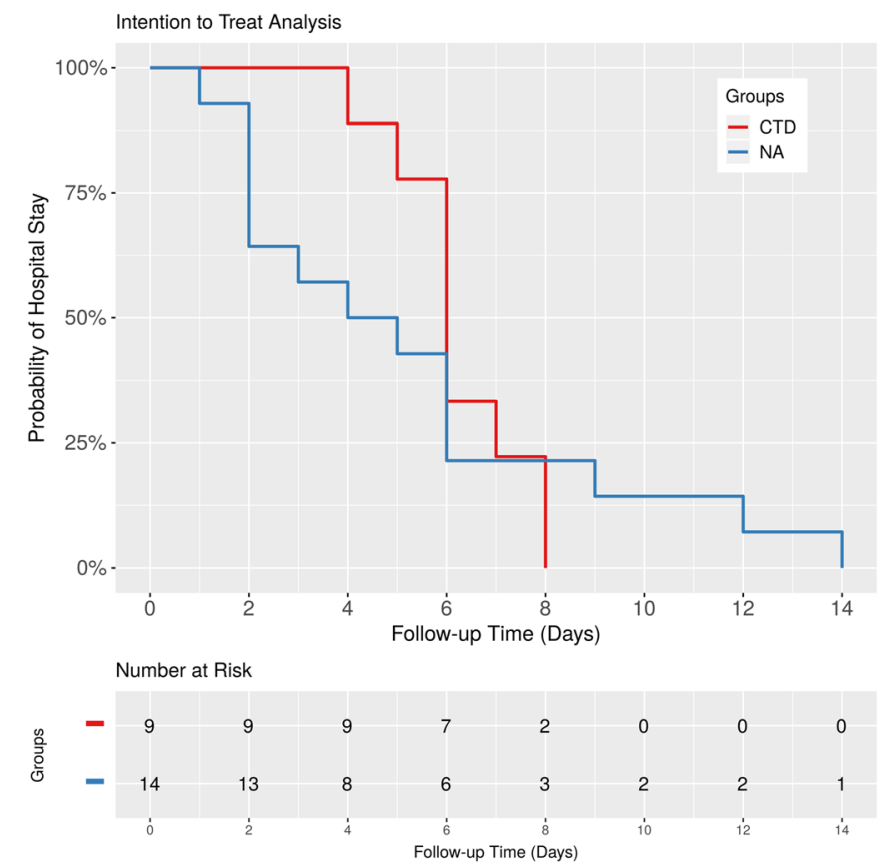

Fig. 2 Length of hospital stay, Kaplan-Meier analysis. Left panel: Intention to treat analysis showed no statistically significant difference in time to event between the two treatment approaches according to the exact log-rank test $(p=0.73)$. Right panel: In contrast, per protocol
CI -5.0 to -2.0 days, $p=0.0012$, exact Wilcoxon-MannWhitney test (Fig. 3, middle panel).

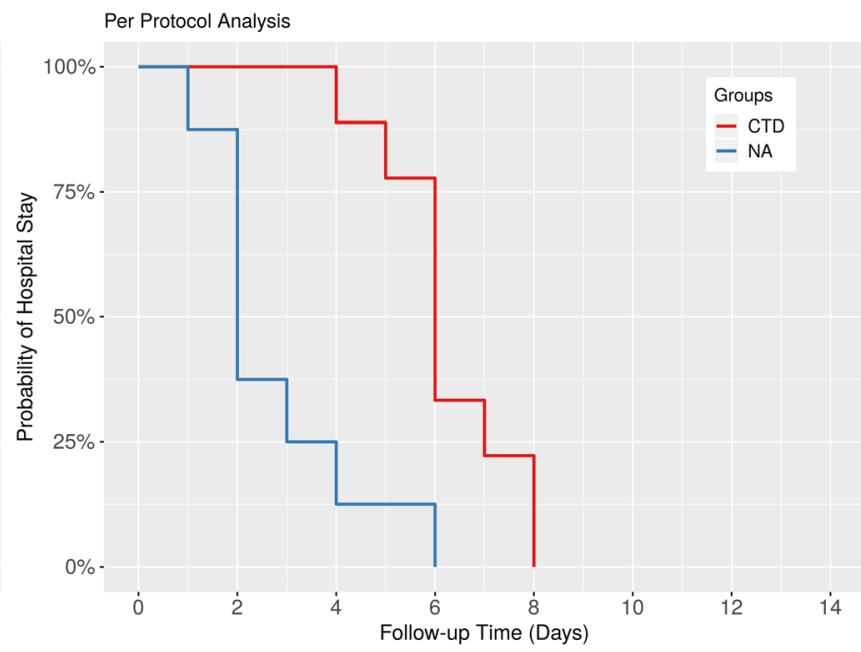

Number at Risk

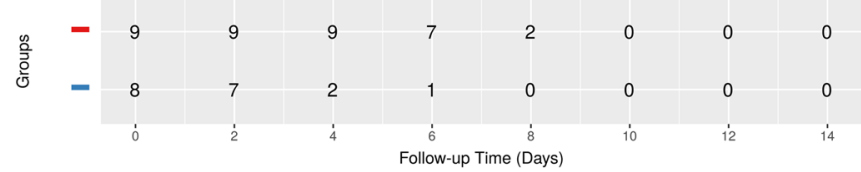

evaluation revealed reduced risk of prolonged hospitalization for successful needle aspiration patients compared with primary chest tube thoracostomy cases ( $p=0.0025$; for details, see text). CTD indicates chest tube drainage, NA stands for needle aspiration 
Fig. 3 Length of hospital stay, median differences with $95 \%$ confidence intervals. Upper panel: Intention to treat analysis showed no statistically significant difference in median difference between the two treatment approaches according to the exact Wilcoxon-Mann-Whitney test $(p=0.17)$. Middle panel: In contrast, per protocol evaluation revealed shortened hospitalization for successful needle aspiration patients compared with primary chest tube drainage $(p=0.0012)$. Lower panel: Unsuccessful primary needle aspiration (i.e., secondary chest tube drainage) did not result in a statistically significant delay in discharge timing $(p=0.28)$. Pr., primary; Sec., secondary; CTD, chest tube drainage; NA, needle aspiration; MD, median difference

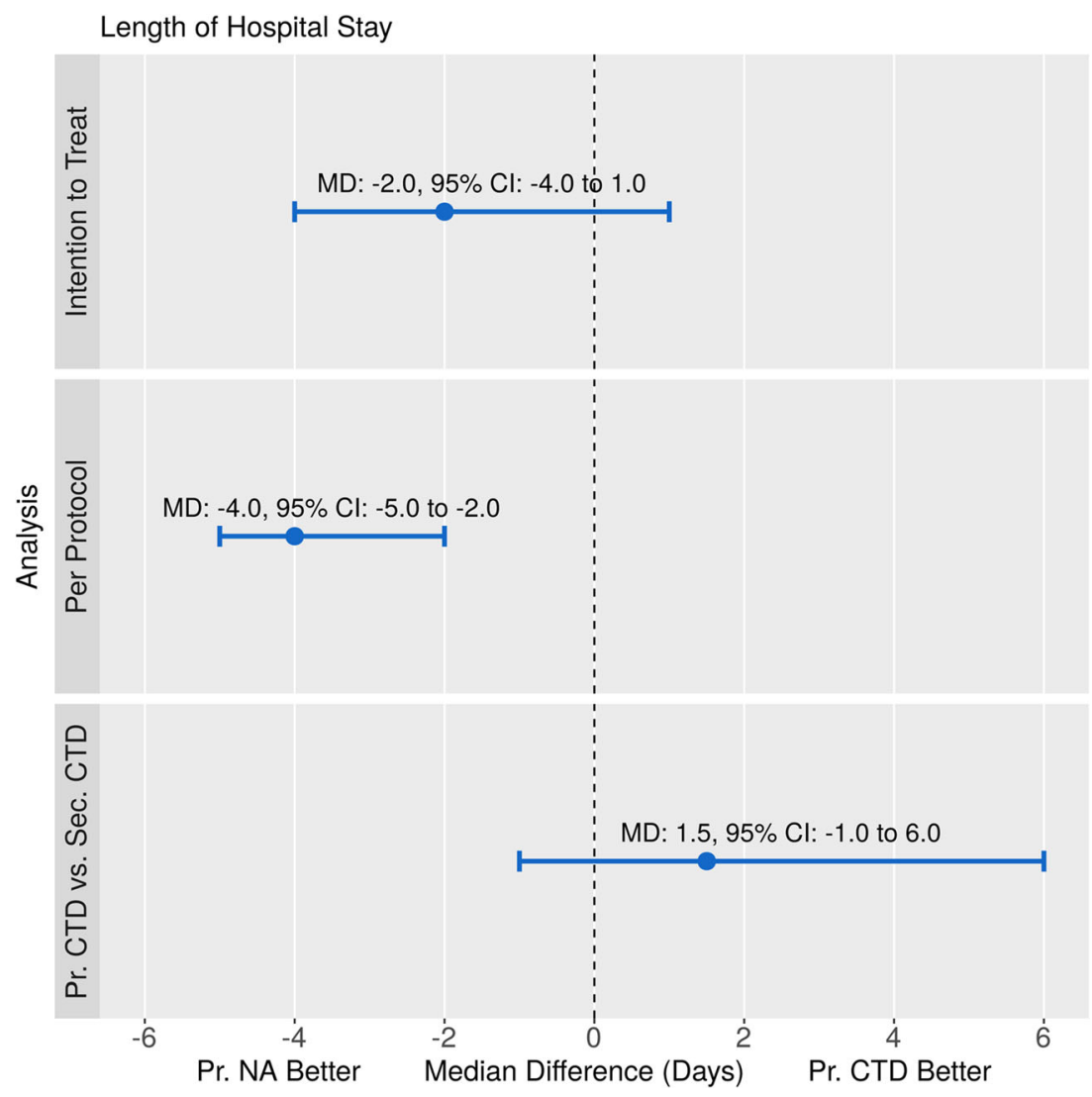

\subsection{Impact of failure of needle aspiration on discharge timing}

Unsuccessful primary needle aspiration did not result in a statistically significant delay in discharge timing as the exact log-rank test $p$ value was 0.10 . Median duration of hospital stays were 7.5 days (IQR 6.0 to 11.25 days) for the failed NA (secondary CTD) and 6.0 days (IQR 6.0 to 7.0 days) for the primary CTD group, the median difference was 1.5 days, $95 \%$ CI -1.0 to 6.0 days, $p=0.28$, exact Wilcoxon-Mann-Whitney test (Fig. 3, lower panel).

\section{Discussion}

\subsection{General considerations}

Iatrogenic pneumothorax requiring intervention is a rare but serious complication of CIED procedures resulting in prolonged hospital stay. It is traditionally treated by largebore chest tube drainage. Nevertheless, it has recently been shown that primary and secondary spontaneous pneumothoraces could also be treated successfully with the novel, less invasive needle aspiration technique [7-9, 12]. It is less traumatic for the patient and may help to shorten hospitalization. Despite the aforementioned data, there is no expert consensus regarding this therapeutic modality: The American College of Chest Physicians Delphi consensus does not advise needle aspiration, but rather supports primary chest tube insertion [14], while the British Thoracic Society guideline recommends needle aspiration as the first-line therapy for primary spontaneous pneumothorax and as an option for small secondary spontaneous pneumothorax [10]. Moreover, to the best of our knowledge, data about its possible role in the treatment of iatrogenic PTX after CIED procedures are completely missing. We hypothesized that, at least in a subset of patients, NA might also be effective in this clinical setting and conducted a preliminary single-center observational efficacy study using a prospective registry. We found that a subset of patients with iatrogenic pneumothorax following CIED procedures may successfully be treated with this technique resulting in shorter hospitalization without the risk of relevant discharge delay in failed cases.

\subsection{Risk of iatrogenic pneumothorax}

The occurrence of PTX is associated with the puncture of the subclavian vein $[1,2]$. Though the operators in this study used the cephalic vein cut-down technique whenever possible to avoid this complication, in a number of cases, puncture of the subclavian vein was inevitable. The occurrence of PTX in our cohort of patients with at least one new subclavian 
venous access was similar to previously reported data in this population, i.e., around $2.4 \%[2,3]$. It is of note, however, that the frequency of PTX may be further reduced using newer techniques like axillary vein cannulation [2] and ultrasound guidance [15]. We found, that PTX was more frequent among women and was associated with lower body mass index (Table 1). This is in accordance with previous findings of large registry-based studies $[1,4-6]$.

\subsection{Procedural success rate, length of hospital stay}

In our cohort, the procedural success rate of NA was $57.1 \%$ (95\% CI 28.9 to $82.3 \%$ ). This is comparable with preceding reports on needle aspiration treatment of primary spontaneous pneumothorax with "immediate" success rates of 59.3 (95\% CI 38.8 to $77.6 \%$ ) to $66.7 \%$ (95\% CI 48.2 to $82.0 \%$ ) [16-18]. Yet, this rate was statistically significantly lower than that of the CTD arm $(100 \%, 95 \%$ CI 66.4 to $100.0 \%, p=0.0481)$. Regarding length of hospital stay, ITT analysis failed to reveal statistical difference between the two concepts (median difference -2.0 days, $95 \% \mathrm{CI}-4.0$ to 1.0 days, $p=0.17$, Fig. 3, upper panel). Despite the statistically non-significant result, an effect size of this magnitude (i.e., a median decrease of 2.0 days) may be of clinical relevance, for which our preliminary study with limited sample size was not powered. Nevertheless, per protocol evaluation of the data shows that with primary NA, nearly $60 \%$ (57.1\%) of the PTX cases could be discharged median 4 days earlier (Fig. 3, middle panel). On the other hand, discharge timing of the patients with failed needle aspiration (42.9\%) was not delayed significantly (in both statistical and clinical sense): the median difference was 1.5 days (Fig. 3, lower panel) corresponding to the procedural protocol (with last control CXR after $24 \mathrm{~h}$ of observation, as described in Sect. 2) and the time needed for adjudication of success and actual discharge. Thus, a considerable number of patients with iatrogenic pneumothorax following cardiac implantable electronic device procedures may be successfully treated with the less invasive needle aspiration resulting in shorter hospitalization without the risk of meaningful discharge delay in unsuccessful cases. Moreover, these findings may raise the possibility that, despite the statistically nonsignificant results of the ITT analysis, the strategy of primary needle aspiration may have a clinical potential relevant effect on discharge timing. Of course, testing this hypothesis requires further studies with larger sample size.

\subsection{Technical considerations}

Prior works pointed out the importance of the correlation between chest wall thickness and body mass index on proper selection of catheter length $[13,19]$. The BMI of our patients was less than $31 \mathrm{~kg} / \mathrm{m}^{2}$ and the pleural space could be reached in every patient with a regular 4.5 -cm-long over-the-needle cannula. Also, the intended puncture track, i.e., second intercostal space in the midclavicular line, should be carefully located to minimize the risk of internal mammary artery injury [13].

\subsection{Limitations}

Our results are based on observational data from a single institutional registry with inherent bias at treatment assignment. The limited sample size did not allow the use of multivariable adjustment or propensity score-based techniques to account for biased covariates. Also, we could not perform uni- and multivariable analysis of possible predictors of procedural success (e.g., size of the pneumothorax) because of lack of appropriate statistical power. Moreover, our pilot study was not powered to detect a clinical potential meaningful effect (i.e., a median decrease of 2.0 days) of primary needle aspiration on length of hospital stay in the intention to treat analysis. Furthermore, being primarily an efficacy analysis, it did not address potential adverse effects related to needle aspiration because of lack of power (no infection, bleeding, or death occurred in any of the treatment arms during the hospital stay). Also, we do not have follow-up efficacy and safety data. Therefore, future multicenter randomized studies or analysis of large national registries using statistical techniques that allow accounting for biased baseline covariates are warranted, particularly given the low incidence rate of this complication.

\section{Conclusion}

Our preliminary data suggest that in a number of patients iatrogenic pneumothorax following cardiac implantable electronic device procedures may be successfully treated with needle aspiration resulting in shorter hospitalization without the risk of meaningful discharge delay in unsuccessful cases.

Funding Information Open access funding provided by Semmelweis University (SU).

\section{Compliance with ethical standards}

The study was conducted in accordance with the amended Declaration of Helsinki. The protocol was approved by the Institutional Ethics Committee on Human Research of the Medical Center Hungarian Defense Forces (approval number: 280-261/2018). All procedures were carried out with the patients' written permission and all of them gave informed consent to participate in the registry.

Conflict of interest The authors declare that they have no conflicts of interest.

Open Access This article is distributed under the terms of the Creative Commons Attribution 4.0 International License (http:// creativecommons.org/licenses/by/4.0/), which permits unrestricted use, distribution, and reproduction in any medium, provided you give 
appropriate credit to the original author(s) and the source, provide a link to the Creative Commons license, and indicate if changes were made.

\section{References}

1. Kirkfeldt RE, Johansen JB, Nohr EA, Moller M, Arnsbo P, Nielsen JC. Pneumothorax in cardiac pacing: a population-based cohort study of 28860 Danish patients. Europace. 2012;14:1132-8. https://doi.org/10.1093/europace/eus054.

2. Kotter J, Lolay G, Charnigo R, Leung S, McKibbin C, Sousa M, et al. Predictors, morbidity, and costs associated with pneumothorax during electronic cardiac device implantation. Pacing Clin Electrophysiol. 2016;39:985-91. https://doi.org/10.1111/pace. 12901.

3. Liu P, Zhou Y-F, Yang P, Gao Y-S, Zhao G-R, Ren S-Y, et al. Optimized axillary vein technique versus subclavian vein technique in cardiovascular implantable electronic device implantation. Chin Med J. 2016;129:2647-51. https://doi.org/10.4103/0366-6999. 193462.

4. Peterson PN, Daugherty SL, Wang Y, Vidaillet HJ, Heidenreich PA, Curtis JP, et al. Gender differences in procedure-related adverse events in patients receiving implantable cardioverter-defibrillator therapy. Circulation. 2009;119:1078-84. https://doi.org/10.1161/ CIRCULATIONAHA.108.793463.

5. Nowak B, Misselwitz B, Erdogan A, Funck R, Irnich W, Israel CW, et al. Do gender differences exist in pacemaker implantation?results of an obligatory external quality control program. Europace. 2010;12:210-5. https://doi.org/10.1093/europace/ eup312.

6. Kirkfeldt RE, Johansen JB, Nohr EA, Jorgensen OD, Nielsen JC. Complications after cardiac implantable electronic device implantations: an analysis of a complete, nationwide cohort in Denmark. Eur Heart J. 2014;35:1186-94. https://doi.org/10.1093/eurheartj/ eht511.

7. Delius RE. Catheter aspiration for simple pneumothorax. Arch Surg. 1989;124:833-6. https://doi.org/10.1001/archsurg.1989. 01410070091018 .

8. Thelle A, Gjerdevik M, SueChu M, Hagen OM, Bakke P. Randomised comparison of needle aspiration and chest tube drainage in spontaneous pneumothorax. Eur Respir J. 2017;49:1601296. https://doi.org/10.1183/13993003.01296-2016.

9. Ramouz A, Lashkari MH, Fakour S, Rasihashemi SZ. Randomized controlled trial on the comparison of chest tube drainage and needle

aspiration in the treatment of primary spontaneous pneumothorax. Pakistan J Med Sci. 2018;34:1369-74. https://doi.org/10.12669/ pjms.346.16126.

10. MacDuff A, Arnold A, Harvey J. Management of spontaneous pneumothorax: British Thoracic Society pleural disease guideline 2010. Thorax. 2010;65:ii18-31. https://doi.org/10.1136/thx.2010. 136986.

11. Collins CD, Lopez A, Mathie A, Wood V, Jackson JE, Roddie ME. Quantification of pneumothorax size on chest radiographs using interpleural distances: regression analysis based on volume measurements from helical CT. Am J Roentgenol. 1995;165:1127-30. https://doi.org/10.2214/ajr.165.5.7572489.

12. Pasquier M, Hugli O, Carron P-N. Needle aspiration of primary spontaneous pneumothorax. N Engl J Med. 2013;368:e24. https:// doi.org/10.1056/NEJMvem1111468.

13. Hecker M, Hegenscheid K, Völzke H, Hinz P, Lange J, Ekkernkamp A, et al. Needle decompression of tension pneumothorax. J Trauma Acute Care Surg. 2016;80:119-24. https://doi. org/10.1097/TA.0000000000000878.

14. Baumann MH, Strange C, Heffner JE, Light R, Kirby TJ, Klein J, et al. Management of spontaneous pneumothorax. Chest. 2001;119: 590-602. https://doi.org/10.1378/chest.119.2.590.

15. Franco E, Rodriguez Muñoz D, Matía R, Hernandez-Madrid A, Carbonell San Román A, Sánchez I, et al. Wireless ultrasoundguided axillary vein cannulation for the implantation of cardiovascular implantable electric devices. J Cardiovasc Electrophysiol. 2016;27:482-7. https://doi.org/10.1111/jce.12917.

16. Andrivet P, Djedaini K, Teboul JL, Brochard L, Dreyfuss D. Spontaneous pneumothorax. Comparison of thoracic drainage vs immediate or delayed needle aspiration. Chest. 1995;108:335-9.

17. Noppen M, Alexander P, Driesen P, Slabbynck H, Verstraeten A. Manual aspiration versus chest tube drainage in first episodes of primary spontaneous pneumothorax. Am J Respir Crit Care Med. 2002;165:1240-4. https://doi.org/10.1164/rccm.200111-078OC.

18. Ayed AK. Aspiration versus tube drainage in primary spontaneous pneumothorax: a randomised study. Eur Respir J. 2006;27:477-82. https://doi.org/10.1183/09031936.06.00091505.

19. Powers WF, Clancy TV, Adams A, West TC, Kotwall CA, Hope WW. Proper catheter selection for needle thoracostomy: a height and weight-based criteria. Injury. 2014;45:107-11. https://doi.org/ 10.1016/j.injury.2013.08.026.

Publisher's note Springer Nature remains neutral with regard to jurisdictional claims in published maps and institutional affiliations. 